\title{
Retrospective Cost Adaptive Flow Control Using a Dielectric Barrier Discharge Actuator
}

\author{
Matthew Fledderjohn, Young-Chang Cho, Jesse B. Hoagg†, Mario Santillo, \\ Wei Shyy $\ddagger$ and Dennis S. Bernstein ${ }^{\S}$ \\ Department of Aerospace Engineering, The University of Michigan, Ann Arbor, MI 48109-2140
}

\begin{abstract}
Retrospective cost adaptive control is applied to low Reynolds number aerodynamics around an SD7003 airfoil with a dielectric barrier discharge (DBD) actuator located near the leading edge. The adaptive control algorithm uses knowledge of Markov parameters to capture information about any nonminimum-phase zeros in the dynamics. In this paper, we explore the impact of the adaptive controller on the aerodynamics under the chord Reynolds numbers of 300 and 60,000, and with both steady and unsteady free-stream conditions. By varying the voltage to the DBD actuator, effective control of unsteady flow structure can be performed to decrease drag and increase lift.
\end{abstract}

\section{Nomenclature}

$\begin{array}{ll}\mathrm{E} & \text { Electric field vector } \\ F_{\text {avg }} & \text { Quasi-steady body force from the DBD actuator } \\ F_{1}, F_{2} & \text { Body forces in the } x_{1} \text { and } x_{2} \text { directions } \\ q_{i} & \text { Electric charge of a species } \\ n_{i} & \text { Particle number density of a species } \\ \rho_{c} & \text { Net charge density } \\ q_{c} & \text { Unit electric charge }\left(=1.6 \times 10^{-19} \mathrm{C}\right) \\ c & \text { Chord length of airfoil } \\ U & \text { Free stream velocity } \\ C_{\mathrm{l}} & \text { Lift coefficient of an airfoil, which is the integral over the surface of the airfoil of } \\ & \text { the normalized pressure force in the direction of lift } \\ C_{\mathrm{d}} & \text { Drag coefficient of an airfoil, which is the integral over the surface of the airfoil of } \\ & \text { the normalized pressure force in the direction of drag } \\ V_{\text {app }} & \text { Voltage applied to the DBD actuator } \\ U t / c & \text { Normalized time in the flow simulation } \\ R e & \text { Reynolds number }\end{array}$

\section{Introduction}

Low Reynolds number flows, specifically flows with Reynolds numbers less than $10^{5}$, are susceptible to flow separation, laminar-turbulent transition, and reattachment, resulting in severe performance losses. ${ }^{1} \mathrm{In}$ particular, micro air vehicles (MAVs) with maximum dimension smaller than $15 \mathrm{~cm}$ and maximum flight speeds of around $10 \mathrm{~m} / \mathrm{s}$ are inherently vulnerable to gusty flight conditions, ${ }^{2}$ and thus require an effective flow control strategy for practical operation. Closed-loop active flow control is an area of active research. Huang et al. employ a closed-loop PID controller based on the variable structure model using dielectric barrier discharge (DBD) actuators to increase the power efficiency in noise reduction. ${ }^{3}$ In Ref. 4, a linear

\footnotetext{
*Graduate student, AIAA Student Member.

${ }^{\dagger}$ Postdoctoral Research Fellow, AIAA Member.

$\ddagger$ Professor, AIAA Fellow.

$\S$ Professor, AIAA Member.
} 
quadratic regulator (LQR) controller augmented with an adaptive control input is applied to perform airfoil maneuvers using symmetric synthetic jets at the trailing edge. Regarding model-based approaches in flow control, control laws based on the proper orthogonal decomposition (POD) method are applied, for example, to control 3D leading edge vorticity ${ }^{5}$ and flow over a shallow cavity. ${ }^{6}$ In addition, adaptive control techniques have been applied to a variety of flow control problems. ${ }^{7-11}$ In particular, Ref. 7,8 use extremum seeking methods to control the drag on a bluff body. In Ref. 9-11, discrete-time adaptive control algorithms are used to control the flow in a two-dimensional channel under a variety of flow conditions, including steady and time-varying flow.

Adaptive control techniques have several characteristics that may make such methods well suited for active flow control. In particular, adaptive controllers tune the feedback gains in response to the true plant dynamics and exogenous signals such as commands and disturbances. In flow control, this is important since the plant dynamics and exogenous signals may change significantly as the flow conditions vary. Furthermore, adaptive controllers generally require limited model information, which is beneficial for flow control where developing high-fidelity models for control purposes is difficult. However, adaptive control laws usually involve restrictive assumptions, such as the plant being passive ${ }^{12}$ or minimum phase. ${ }^{13}$ In addition, sensitivity to unmodeled dynamics and sensor noise ${ }^{14}$ as well as unfavorable transient performance ${ }^{15}$ are common issues related to stability and performance in adaptive control.

In this paper, we use a discrete-time adaptive control method for flow control. In Ref. 13, a discrete-time adaptive controller algorithm is presented for plants under a minimum-phase assumption. In Ref. 16, the authors invoke the same minimum phase assumption to develop an adaptive control method for command following and disturbance rejection of sinusoids where the command and disturbance are unmeasured and the spectrum of those signals is unknown. A discrete-time adaptive control law that is known numerically to be effective on nonminimum-phase plants is developed in Ref. 17.

In Ref. 18, the adaptive control method of Ref. 17 is used to control the lift in low Reynolds number aerodynamics by using a DBD actuator. The results of Ref. 18 demonstrate the ability to modify the attached or moderately separated flow over an SD7003 airfoil with minimal modeling information. More specifically, the method requires some information on the Markov parameters of the plant, which are extracted by parameter estimation from numerical simulations. The results of Ref. 18 use a Reynolds number based on the airfoil chord length set at 60,000 .

In the present paper, we extend the work of Ref. 18 by applying the adaptive control technique based on retrospective cost optimization developed in Refs. 19,20. Retrospective cost adaptive control (RCAC) modifies the sensor measurements using the difference between the actual past control inputs and the recomputed past control inputs based on the current controller parameters. In Refs. 19,20, RCAC is shown to be effective for unstable nonminimum-phase systems as long as a sufficient number of Markov parameters are available (through modeling or parameter estimation) to approximate the location of the nonminumum-phase zeros (if any) of the system.

The emphasis of this paper is to explore the interplay between the aerodynamics and the control strategy presented in Ref. 19,20. Nevertheless, there are several issues with applying DBD actuators to low Reynolds number flyers. In particular, DBD actuator performance is significantly impacted by the actuator location and geometry, ${ }^{21}$ the actuator's wave form and frequency, ${ }^{22}$ as well as the dielectric material. ${ }^{23}$ While these issues are being explored through methods such as the use of surrogate models for DBD actuators, ${ }^{24}$ this paper focuses on the implications of the adaptive flow control method and does not address these other topics.

The contents of this paper are as follows. In Section II, we present the numerical flow model. Section III introduces the adaptive controller used to control the free stream flow. In Section IV, we discuss identification of the Markov parameters required by the adaptive controller. Simulation results are presented in Section V.

\section{Numerical Flow Model}

In this paper, flow fields are analyzed by solving the Reynolds-averaged Navier-Stokes equations using the pressure-based solver Loci-STREAM ${ }^{25}$ and adopting a parallelized unstructured finite volume code. Since the ion and electron states are non-equilibrium and the ion temperature is comparable to the neutral fluid, the neutral fluid is treated as being isothermal. Considering the time scale disparity between the flow and the radio frequency $(\mathrm{RF})$ actuator operation, the force acting on the neutral fluid is assumed to be a quasi-steady 
body force. The body force felt by the neutral flow is equivalent to the Lorentz force acting on the net charge density. For the unsteady operation of the actuator only the amplitude variation of the operation voltage with time scales much larger than the RF operation is considered. The relevant conservation equations are

$$
\sum_{j=1}^{2} \frac{\partial u_{j}}{\partial x_{j}}=0
$$

and for $i=1,2$,

$$
\frac{\partial u_{i}}{\partial t}+\sum_{j=1}^{2} \frac{\partial\left(u_{i} u_{j}\right)}{\partial x_{j}}=\frac{F_{i}}{\rho}-\frac{1}{\rho} \frac{\partial p}{\partial x_{i}}+\sum_{j=1}^{2} \frac{\partial}{\partial x_{j}}\left[\left(\nu+\nu_{T}\right) \frac{\partial u_{i}}{\partial x_{j}}\right],
$$

where, for $i=1,2, F_{i}=E_{i} \sum_{k} q_{k} n_{k}, u_{1}$ is the velocity in the $x_{1}$ direction, $u_{2}$ is the velocity in the $x_{2}$ direction, $\rho$ is air density, $p$ is pressure, $\nu$ is the kinematic viscosity, $\nu_{T}$ is the eddy viscosity, $E_{1}$ is the electric field strength in the $x_{1}$ direction, and $E_{2}$ is the electric field strength in the $x_{2}$ direction.

For the turbulence closure, Menter's SST turbulence model is used. ${ }^{26}$ For simplicity, the laminarturbulent transition model is not applied in this paper. For $i=1,2$, Mentor's SST turbulence model is given by

$$
\begin{aligned}
& \frac{\partial k}{\partial t}+\sum_{i=1}^{2} \frac{\partial\left(u_{i} k\right)}{\partial x_{i}}=-\sum_{j=1}^{2} \sum_{i=1}^{2} \frac{\tau_{i, j}}{\rho} \frac{\partial u_{j}}{\partial x_{i}}-\beta^{*} \omega k+\sum_{i=1}^{2} \frac{\partial}{\partial x_{i}}\left[\left(\nu+\sigma_{k} \nu_{T}\right) \frac{\partial k}{\partial x_{i}}\right] \\
& \frac{\partial \omega}{\partial t}+\sum_{i=1}^{2} \frac{\partial\left(u_{i} \omega\right)}{\partial x_{i}}=-\sum_{j=1}^{2} \sum_{i=1}^{2} \gamma^{*} \frac{\tau_{i, j}}{\rho \nu_{T}} \frac{\partial u_{j}}{\partial x_{i}}-\beta \omega^{2}+\sum_{i=1}^{2} \frac{\partial}{\partial x_{i}}\left[\left(\nu+\sigma_{\omega} \nu_{T}\right) \frac{\partial \omega}{\partial x_{i}}\right]+\sum_{i=1}^{2} 2\left(1-\bar{F}_{1}\right) \frac{\sigma_{\omega, 2}}{\omega} \frac{\partial k}{\partial x_{i}} \frac{\partial \omega}{\partial x_{i}}
\end{aligned}
$$

where

$$
\tau_{i, j}=\rho \nu_{T}\left(\frac{\partial u_{i}}{\partial x_{j}}+\frac{\partial u_{j}}{\partial x_{i}}\right)-\frac{2}{3} \rho k \delta_{i, j}, \quad \nu_{T}=\frac{a_{1} k}{\max \left(a_{1} \omega,\left\|\nabla \times u_{i}\right\| \bar{F}_{2}\right)},
$$

where $k$ and $\omega$ are the turbulence kinetic energy and specific rate of dissipation of the turbulence kinetic energy, respectively, and $\delta_{i, j}$ is the Kronecker delta. The values of the coefficients $a_{1}, \beta, \beta^{*}, \gamma^{*}, \sigma_{k}, \sigma_{\omega}, \sigma_{\omega, 2}$, $\bar{F}_{1}$, and $\bar{F}_{2}$ as well as the boundary conditions for the turbulence model are given in Ref. 26.

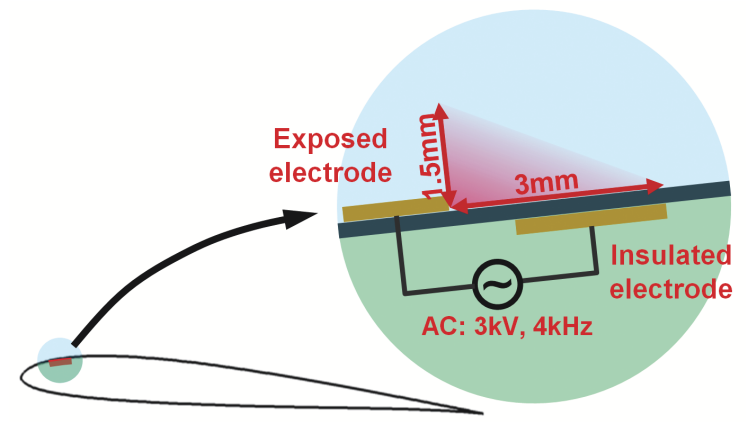

Figure 1. Simplified DBD model geometry

The DBD actuator model is a simplified model with linear electric field and constant net charge density. $^{27,28}$ As presented in Figure 1, this model prescribes localized body forces in a triangular plasma region bounded by two electrodes and dielectric surface. The electric field distribution inside the plasma region is approximated by the spatially linear relations

$$
E_{1}=\frac{|\mathrm{E}| k_{2}}{\sqrt{k_{1}^{2}+k_{2}^{2}}}, \quad E_{2}=\frac{|\mathrm{E}| k_{1}}{\sqrt{k_{1}^{2}+k_{2}^{2}}}
$$

where

$$
|\mathrm{E}|=E_{0}-k_{1} x_{1}-k_{2} x_{2}, \quad E_{0}=\frac{V_{\text {app }}}{d},
$$


where $d$ is the insulator thickness, and $k_{1}$ and $k_{2}$ are the linearized slopes of the electric field in the $x_{1}$ and $x_{2}$ directions, respectively. This is a solution of Gauss' equation with the constant net charge density assumption. In (4) the maximum electric field intensity $E_{0}$ is defined based on the applied voltage amplitude, and the slopes $k_{1}$ and $k_{2}$ of the electric field attenuation away from the exposed electrode and dielectric surface, are set to allow the breakdown voltage at the boundary with the minimum electric field strength. As shown in Ref. 28, this analytical-empirical model represents a time-averaged body force component acting on the fluid, given by

$$
F_{\text {avg }}\left(x_{1}, x_{2}, t\right)=\rho_{c} q_{c} \delta\left(x_{1}, x_{2}\right) f_{v} \Delta t \mathrm{E}\left(x_{1}, x_{2}, t\right),
$$

where $f_{v}$ is the AC frequency of the voltage applied to the DBD actuator and $\Delta t$ is the discharge duty cycle. Furthermore, since the constant charge density $\rho_{c}$ with unit charge $q_{c}$ is present only inside the plasma region, $\delta\left(x_{1}, x_{2}\right)$ is set to 0 or 1 depending on the position. The discharge duty cycle is the portion of time during which effective force generation occurs in each operation cycle. For the purpose of the control input the applied voltage to the electrode is changed depending on the controller command, resulting in a time-varing body force. Since the simplified DBD model is based on the quasi-steady assumption using the time scale disparity, the unsteady control input is meaningful when its timescale lies between those of low Reynolds number flow and plasma operation. This simplified DBD model presents good agreement with experimental data in terms of force generation ${ }^{28}$ and maximum induced flow velocity. ${ }^{29}$ In order to assess the resultant performance of the DBD actuator and controller, a single co-flow directional DBD actuator with voltage amplitude modulation according to the controller output is used.

\section{Controller Construction}

Consider the multi-input, multi-output linear discrete-time system

$$
\begin{aligned}
x(k+1) & =A x(k)+B u(k)+D_{1} w(k), \\
y(k) & =C x(k)+D_{2} w(k), \\
z(k) & =E_{1} x(k)+E_{0} w(k),
\end{aligned}
$$

where $x(k) \in \mathbb{R}^{n}$ is the state, $y(k) \in \mathbb{R}^{l_{y}}$ is the measurement, $z(k) \in \mathbb{R}^{l_{z}}, u(k) \in \mathbb{R}^{l_{u}}$ is the performance, $w(k) \in \mathbb{R}^{l_{w}}$ is the exogenous command and or disturbance signal, and $k \geq 0$. We present an adaptive output feedback controller under which the performance variable $z$ is minimized in the presence of the exogenous signal $w$, which could be a disturbance, command, or both. In this paper, we use an adaptive feedback controller to minimize the drag force acting on the airfoil under both undisturbed inlet flow conditions and in the presence of a sinusoidal disturbance to the inlet flow.

In this section, we summarize the adaptive control algorithm as presented in Ref. 20 for the general control problem given by (6)-(8). We use a strictly proper time-series controller of order $n_{\mathrm{c}}$, such that the control $u(k)$ is given by

$$
u(k)=\sum_{i=1}^{n_{\mathrm{c}}} M_{i}(k) u(k-i)+\sum_{i=1}^{n_{\mathrm{c}}} N_{i}(k) y(k-i),
$$

where, for all $i=1, \ldots, n_{\mathrm{c}}, M_{i} \in \mathbb{R}^{l_{u} \times l_{u}}$ and $N_{i} \in \mathbb{R}^{l_{u} \times l_{y}}$ are given by the adaptive law presented below. The control can be expressed as

$$
u(k)=\theta(k) \phi(k),
$$

where

$$
\theta(k) \triangleq\left[\begin{array}{llllll}
N_{1}(k) & \cdots & N_{n_{\mathrm{c}}}(k) & M_{1}(k) & \cdots & M_{n_{\mathrm{c}}}(k)
\end{array}\right]
$$


is the controller parameter matrix, and the regressor vector $\phi(k)$ is given by

$$
\phi(k) \triangleq\left[\begin{array}{c}
y(k-1) \\
\vdots \\
y\left(k-n_{\mathrm{c}}\right) \\
u(k-1) \\
\vdots \\
u\left(k-n_{\mathrm{c}}\right)
\end{array}\right] \in \mathbb{R}^{n_{\mathrm{c}}\left(l_{u}+l_{y}\right)} .
$$

For positive integers $p$ and $\mu$, we define the extended measurement vector $Y(k)$, the extended performance vector $Z(k)$, and the extended control vector $U(k)$ by

$$
Y(k) \triangleq\left[\begin{array}{c}
y(k) \\
\vdots \\
y(k-p+1)
\end{array}\right], \quad Z(k) \triangleq\left[\begin{array}{c}
z(k) \\
\vdots \\
z(k-p+1)
\end{array}\right], \quad U(k) \triangleq\left[\begin{array}{c}
u(k) \\
\vdots \\
u\left(k-p_{\mathrm{c}}+1\right)
\end{array}\right],
$$

where $p_{\mathrm{c}} \triangleq \mu+p$.

From (10), it follows that the extended control vector $U(k)$ can be written as

$$
U(k) \triangleq \sum_{i=1}^{p_{\mathrm{c}}} L_{i} \theta(k-i+1) \phi(k-i+1),
$$

where

$$
L_{i} \triangleq\left[\begin{array}{c}
0_{(i-1) l_{u} \times l_{u}} \\
I_{l_{u}} \\
0_{\left(p_{\mathrm{c}}-i\right) l_{u} \times l_{u}}
\end{array}\right] \in \mathbb{R}^{p_{\mathrm{c}} l_{u} \times l_{u}}
$$

Next, define the retrospective performance vector

$$
\hat{Z}(\hat{\theta}(k), k) \triangleq Z(k)-\bar{B}_{z u}(U(k)-\hat{U}(k)),
$$

where $\hat{U}(k) \triangleq \sum_{i=1}^{p_{\mathrm{c}}} L_{i} \hat{\theta}(k) \phi(k-i+1), \hat{\theta}(k) \in \mathbb{R}^{l_{u} \times n_{\mathrm{c}}\left(l_{u}+l_{y}\right)}$, and the block-Toeplitz control matrix $\bar{B}_{z u}$ is given by $(31)$ below. Note that $\hat{Z}(\hat{\theta}(k), k)$ is obtained by modifying the performance variable $Z(k)$ based on the difference between the actual past control inputs $U(k)$ and the recomputed past control inputs $\hat{U}(k)$ assuming that the controller parameters $\hat{\theta}(k)$ had been used in the past. Thus, $\hat{Z}(\hat{\theta}(k), k)$ may be interpreted as an approximation of the performance had the controller parameters $\hat{\theta}(k)$ been used in the past.

Taking the vec of (15) yields

$$
\hat{Z}(\hat{\theta}(k), k)=f(k)+D(k) \operatorname{vec} \hat{\theta}(k),
$$

where

$$
\begin{aligned}
f(k) & \triangleq Z(k)-\bar{B}_{z u} U(k), \\
D(k) & \triangleq \sum_{i=1}^{p_{\mathrm{c}}} \phi^{\mathrm{T}}(k-i+1) \otimes \bar{B}_{z u} L_{i} .
\end{aligned}
$$

Note that

$$
\hat{U}(k)=\sum_{i=1}^{p_{\mathrm{c}}} L_{i} \hat{\theta}(k) \phi(k-i+1)=M(k) \operatorname{vec} \hat{\theta}(k)
$$

where $M(k) \triangleq \sum_{i=1}^{p_{c}} \phi^{\mathrm{T}}(k-i+1) \otimes L_{i}$. 
Now, consider the retrospective cost function

$$
\begin{aligned}
\hat{J}(k) \triangleq & \hat{Z}^{\mathrm{T}}(\hat{\theta}(k), k) R_{1}(k) \hat{Z}(\hat{\theta}(k), k)+\hat{U}^{\mathrm{T}}(\hat{\theta}(k), k) R_{2}(k) \hat{U}(\hat{\theta}(k), k) \\
& +\operatorname{tr}\left[(\hat{\theta}(k)-\theta(k))^{\mathrm{T}} R_{3}(k)(\hat{\theta}(k)-\theta(k))\right],
\end{aligned}
$$

where $R_{1}(k)=R_{1}^{\mathrm{T}}(k)>0, R_{2}(k) \geq 0$, and $R_{3}(k)=R_{3}^{\mathrm{T}}(k)>0$. Substituting (16) and (17) into (18) yields

$$
\hat{J}(k)=c(k)+b^{\mathrm{T}}(k) \operatorname{vec} \hat{\theta}(k)+(\operatorname{vec} \hat{\theta}(k))^{\mathrm{T}} A(k) \operatorname{vec} \hat{\theta}(k),
$$

where

$$
\begin{aligned}
A(k) & \triangleq D^{\mathrm{T}}(k) R_{1}(k) D(k)+M^{\mathrm{T}}(k) R_{2}(k) M(k)+R_{3}(k) \otimes I_{n_{\mathrm{c}}(l u+l y)}, \\
b(k) & \triangleq 2 D^{\mathrm{T}}(k) R_{1}(k) f(k)-2 R_{3}(k) \otimes I_{n_{\mathrm{c}}(l u+l y)} \operatorname{vec} \theta(k), \\
c(k) & \triangleq f^{\mathrm{T}}(k) R_{1}(k) f(k)+\operatorname{tr}\left[\theta^{\mathrm{T}}(k) R_{3}(k) \theta(k)\right] .
\end{aligned}
$$

Since $A(k)$ is positive definite, $\hat{J}(k)$ has the strict global minimizer

$$
\hat{\theta}(k)=-\frac{1}{2} \operatorname{vec}^{-1}\left(A^{-1}(k) b(k)\right) .
$$

Thus, the update law is given by

$$
\theta(k+1)=\hat{\theta}(k) .
$$

For all future discussion, we specialize (19)-(21) with

$$
R_{1}(k) \triangleq I_{p l_{z}}, \quad R_{2}(k) \triangleq 0_{p_{c} l_{u}}, \quad R_{3}(k) \triangleq \alpha(k) I_{l_{u}},
$$

where the learning rate $\alpha(k)>0$ affects the transient performance and the convergence speed of the adaptive control algorithm. In this case,

$$
\begin{aligned}
A(k) & =D^{\mathrm{T}}(k) D(k)+\alpha(k) I, \\
b(k) & =2 D^{\mathrm{T}}(k) f(k)-2 \alpha(k) \operatorname{vec} \theta(k), \\
c(k) & =f^{\mathrm{T}}(k) f(k)+\alpha(k) \operatorname{tr}\left[\theta^{\mathrm{T}}(k) \theta(k)\right] .
\end{aligned}
$$

The novel feature of the adaptive control algorithm (10) and (22)-(26) is the use of the retrospective correction filter (RCF) (15). RCF provides an inner loop to the adaptive control law by modifying the performance variable $Z(k)$ based on the difference between the actual past control inputs $U(k)$ and the recomputed past control inputs $\hat{U}(k)$ assuming that the current controller parameters $\hat{\theta}(k)$ had been used in the past.

The adaptive controller (10) and (22)-(26) requires limited model information of the plant (6)-(8); however, the controller does require knowledge of $\bar{B}_{z u}$. The $\bar{B}_{z u}$ matrix is constructed from the plant's Markov parameters. Consider the $\mu$-MARKOV model from $u$ to $z$ given by

$$
z(k)=-\sum_{i=1}^{n} \alpha_{\mu, i} z(k-\mu-i)+\sum_{i=d}^{\mu} H_{i} u(k-i)+\sum_{i=1}^{n} \beta_{\mu, i} u(k-\mu-i),
$$

where $\alpha_{\mu, i} \in \mathbb{R}, \beta_{\mu, i} \in \mathbb{R}^{l_{z} \times l_{u}}, H_{i} \in \mathbb{R}^{l_{z} \times l_{u}}$, and $\mu \geq d$. Equation (27) can be equivalently represented as the $\mu$-MARKOV transfer function

$$
G_{\mu, z u}(\mathbf{z})=\frac{1}{p_{\mu}(\mathbf{z})}\left(H_{d} \mathbf{z}^{\mu+n-d}+\cdots+H_{\mu} \mathbf{z}^{n}\right)+\frac{1}{p_{\mu}(\mathbf{z})}\left(\beta_{\mu, 1} \mathbf{z}^{n-1}+\cdots+\beta_{\mu, n}\right),
$$

where $p_{\mu}(\mathbf{z}) \triangleq \mathbf{z}^{\mu+n}+\alpha_{\mu, 1} \mathbf{z}^{n-1}+\cdots+\alpha_{\mu, n}$. This system representation is nonminimal, overparameterized, and has order $n+\mu$. Note that the coefficients of the terms $\mathbf{z}^{n+\mu-1}$ through $\mathbf{z}^{n}$ in the denominator are zero. 
The Laurent series expansion of $G_{z u}(\mathbf{z})$ about $\mathbf{z}=\infty$ is

$$
G_{z u}(\mathbf{z})=\sum_{i=d}^{\infty} \mathbf{z}^{-i} H_{i}
$$

Truncating the numerator and denominator of (28) is equivalent to the truncated Laurent series expansion of $G_{z u}(\mathbf{z})$ about $\mathbf{z}=\infty$. Thus, the truncated Laurent series expansion of $G_{z u}(\mathbf{z})$ is

$$
\bar{G}_{\mu, z u}(\mathbf{z}) \triangleq \sum_{i=d}^{\mu} \mathbf{z}^{-i} H_{i}
$$

The resulting block-Toeplitz control matrix $\bar{B}_{z u} \in \mathbb{R}^{p l_{z} \times p_{\mathrm{c}} l_{u}}$ is

$$
\bar{B}_{z u} \triangleq\left[\begin{array}{ccccc}
0_{l_{z} \times d l_{u}} & H_{d} & \cdots & H_{\mu} & 0_{l_{z} \times l_{u}(p-1)} \\
\vdots & & \ddots & \ddots & \\
0_{l_{z} \times d l_{u}} & 0_{l_{z} \times l_{u}(p-1)} & H_{d} & \cdots & H_{\mu}
\end{array}\right] .
$$

The leading zeros in the first row of $\bar{B}_{z u}$ account for the nonzero relative degree $d$. The advantage in constructing $\bar{B}_{z u}$ using the Markov parameters $H_{d}, \ldots, H_{\mu}$ as opposed to using all of the numerator coefficients of $G_{\mu, z u}$ is ease of identification. In the case with $z=y$, using the retrospective performance variable $\hat{z}$ in place of $y$ in the regressor vector (11) results in faster convergence of the adaptive algorithm.

Note that for a single-input, single-output system, some of the roots of the polynomial

$$
H(\mathbf{z}) \triangleq H_{d} \mathbf{z}^{\mu-d}+H_{d+1} \mathbf{z}^{\mu-d-1}+\cdots+H_{\mu-1} \mathbf{z}+H_{\mu}
$$

can be shown to approximate the nonminimum-phase zeros from $u$ to $z$ that lie outside of a circle in the complex plane centered at the origin with radius equal to the spectral radius of $A$. Thus, knowledge of $H_{d}, \ldots, H_{\mu}$ encompasses knowledge of the nonminimum-phase zeros from $u$ to $z$ that lie outside of the spectral radius of $A$. In fact, if the transfer function from $u$ to $z$ is minimum phase, then we choose $\mu=d$, which requires knowledge of only a single Markov parameter, namely, $H_{d}$. The minimum-phase case with $z=y$ is considered in Ref. 16 using a gradient-based adaptive law rather than the adaptive law (22)-(26). Under the minimum-phase assumption, Ref. 16 proves asymptotic convergence of $z$ to zero.

\section{Flow Model Parameters and Identification of Markov Parameters}

In this section, we describe the flow model parameters used in this paper and describe the estimation method used to obtain the Markov parameters required by the adaptive controller (10) and (22)-(26). Flow simulations are performed for the airfoil SD7003 with a Reynolds number of 300 and a 15-degree angle of attack. Under these flow conditions, the flow on the upper surface of the airfoil is separated, as shown in Figure 2. The DBD actuator is located at 20 percent of the chord length from the leading edge with the co-flow orientation. The DBD actuator has a nominal voltage of $1 \mathrm{kV}$. This nominal voltage decreases the size of separated flow region, but the the separated flow topology still exists under the nominal actuation, as shown in Figure 3. It is known that there are multiple modes in atmospheric gas discharge,${ }^{30}$ and the transitions between these modes depend on various parameters, ${ }^{31}$ particularly the voltage applied to the DBD actuator. However, typical DBD actuators use voltages up to $30 \mathrm{kV} .{ }^{21,32}$ In this paper, we do not implement bounds on the operational voltage of the DBD actuator.

To estimate the Markov parameters of the flow model, and thus obtain $\bar{B}_{z u}$, the DBD actuator is excited by an impulse command and two system responses are measured, namely the form drag (i.e., the drag coefficient $C_{\mathrm{d}}$ ) and the form lift (i.e., the lift coefficient $C_{\mathrm{l}}$ ). More specifically, the flow model is excited by a $3-\mathrm{kV}$ impulse voltage applied to the DBD actuator; this impulse was numerically determined to sufficiently excite the flow dynamics of interest.

The flow simulation is numerically discretized and run with a normalized time step of 0.05 . In the next section, we implement the adaptive controller (10) and (22)-(26) using a time step equal to ten flow simulation time steps. Therefore, we measure the form drag and form lift impulse responses every ten flow simulation time steps to estimate the Markov parameters of the system from the DBD actuator voltage to the form drag and to the form lift, respectively. 


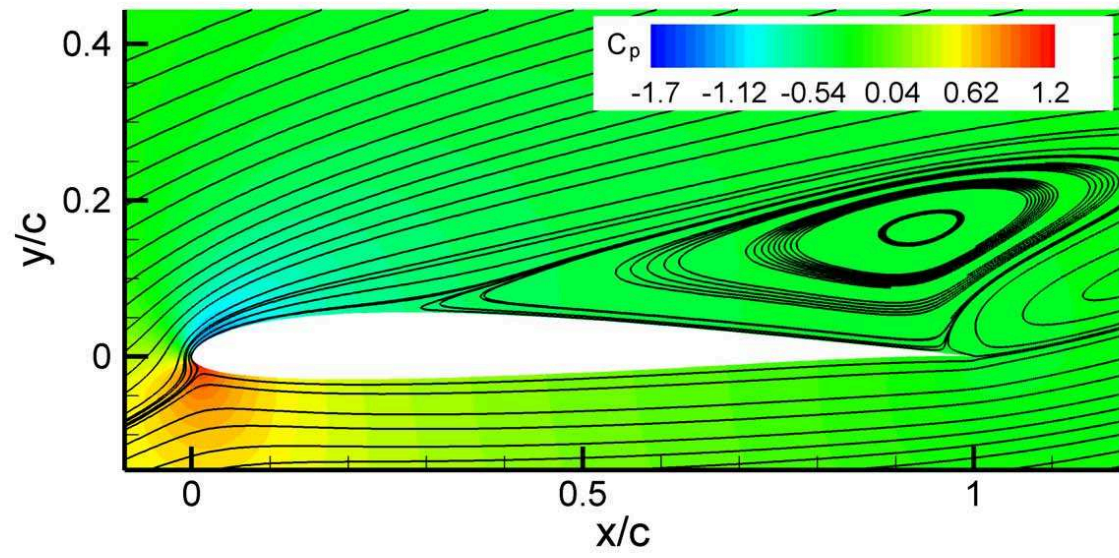

Figure 2. For a steady free-stream flow with $R e=300$, the flow field without actuation shows separation on the upper surface of the airfoil.

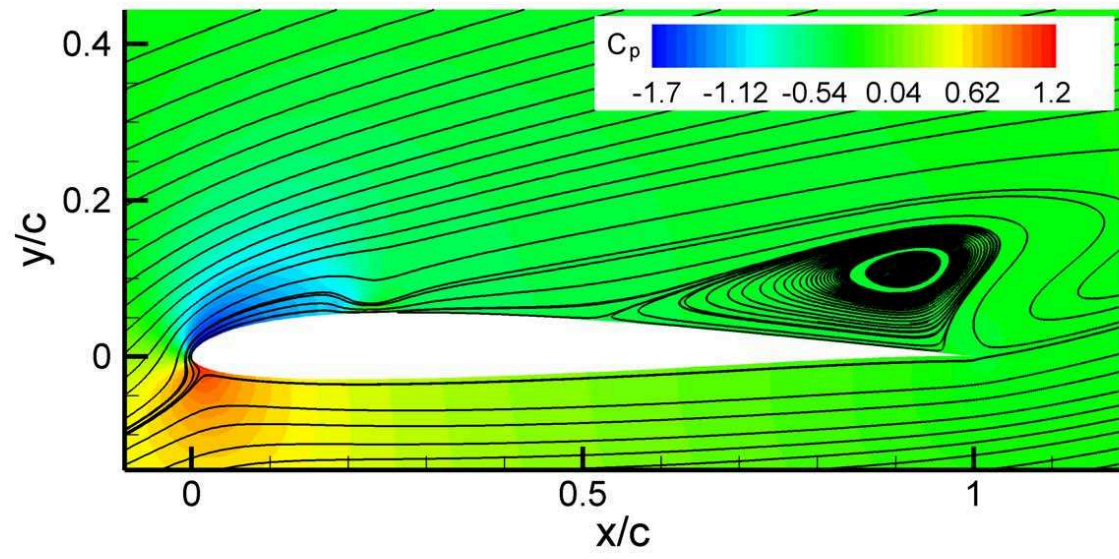

Figure 3. For a steady free-stream flow with $R e=300$, a $1 \mathrm{kV}$ nominal DBD actuator voltage decreases the size of the separated flow region on the upper surface of the airfoil; however, the flow on the upper surface of the airfoil is still separated. 
Figure 4 shows the impulse response of $C_{\mathrm{d}}$. Furthermore, Figure 4 shows the Markov parameters estimated from the impulse response of $C_{\mathrm{d}}$. In particular, we estimate the first 50 Markov parameters from the impulse response. These Markov parameters can be used to construct $\bar{B}_{z u}$ when $C_{\mathrm{d}}$ is used as the performance variable $z(k)$ in the adaptive controller (10) and (22)-(26). Note that the impulse response decays to zero because the open-loop flow dynamics are asymptotically stable. Figure 5 shows the roots of the Markov parameter polynomial (32) with $\mu=50$. Note that all of the roots of (32) lie within the unit circle. Thus, it can be inferred that the linearized discrete-time system from $V_{\text {app }}$ to $C_{\mathrm{d}}$ is minimum phase.

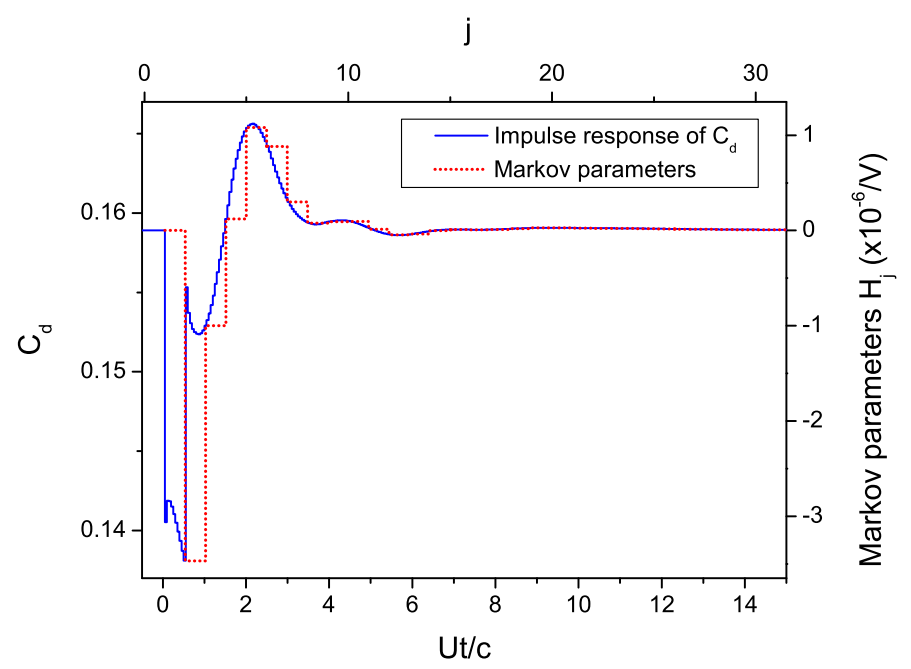

Figure 4. The Markov parameters from $V_{\text {app }}$ to $C_{\mathrm{d}}$ are estimated from the response to a 3-kV impulse under a steady free-stream flow with $R e=300$.

Figure 6 shows the impulse response and estimated Markov parameters using $C_{1}$ as the output. In particular, we estimate the first 50 Markov parameters from the impulse response. These Markov parameters can be used to construct $\bar{B}_{z u}$ when $C_{1}$ is used as the performance variable $z(k)$ in the adaptive controller (10) and (22)-(26). Note that the impulse response decays to zero because the open-loop flow dynamics are asymptotically stable. Figure 5 shows the roots of the Markov parameter polynomial (32) with $\mu=50$. Note that one root of (32) lies outside of the unit circle. Thus, it can be inferred that the linearized discrete-time system from $V_{\text {app }}$ to $C_{1}$ has one nonminimum-phase zero.

The aerodynamic forces acting on the airfoil are composed of a friction force and a pressure force. The flow separation shown in Figure 3 for a high-angle-of-attack airfoil results in significant form drag. Therefore, in the next section, we generally adopt the control objective of minimizing the drag coefficient, and thus reattaching the flow on the upper side of the airfoil. More specifically, the drag coefficient is used in the next section as the performance variable $z(k)$ and the measurement variable $y(k)$ unless otherwise noted.

\section{Results and Discussion}

\section{V.A. Minimization of $C_{\mathrm{d}}$ without inlet disturbance}

In this example, $C_{\mathrm{d}}$ is selected to be the performance variable and $\bar{B}_{z u}$ is constructed using the 50 Markov parameters identified in Figure 4. The learning rate $\alpha=500$ is selected to enforce slow adaptation. Slow adaptation helps to prevent large transient behavior in the flow field; however, it also reduces the speed with which the closed-loop converges to steady-state performance minimization. Decreasing $\alpha$ speeds up adaptation but results in larger transient behavior. Figure 8 shows the closed-loop time histories for $C_{\mathrm{d}}, C_{\mathrm{l}}$, and $V_{\text {app }}$ when the adaptive controller (10) and (22)-(26) is implemented in the feedback loop with $n_{\mathrm{c}}=10$. The nominal drag coefficient is approximately 0.16. The adaptive controller is turned on at $U t / c=4$ and by $U t / c=400, C_{\mathrm{d}}$ converges to zero. At this steady-state condition, $C_{\mathrm{l}}=1$ and $V_{\text {app }}=50 \mathrm{kV}$.

Figure 9 shows the same closed-loop response where a higher-order adaptive controller, that is $n_{\mathrm{c}}=50$, is implemented in the feedback loop. In this case, the closed-loop system converges to the same steady- 


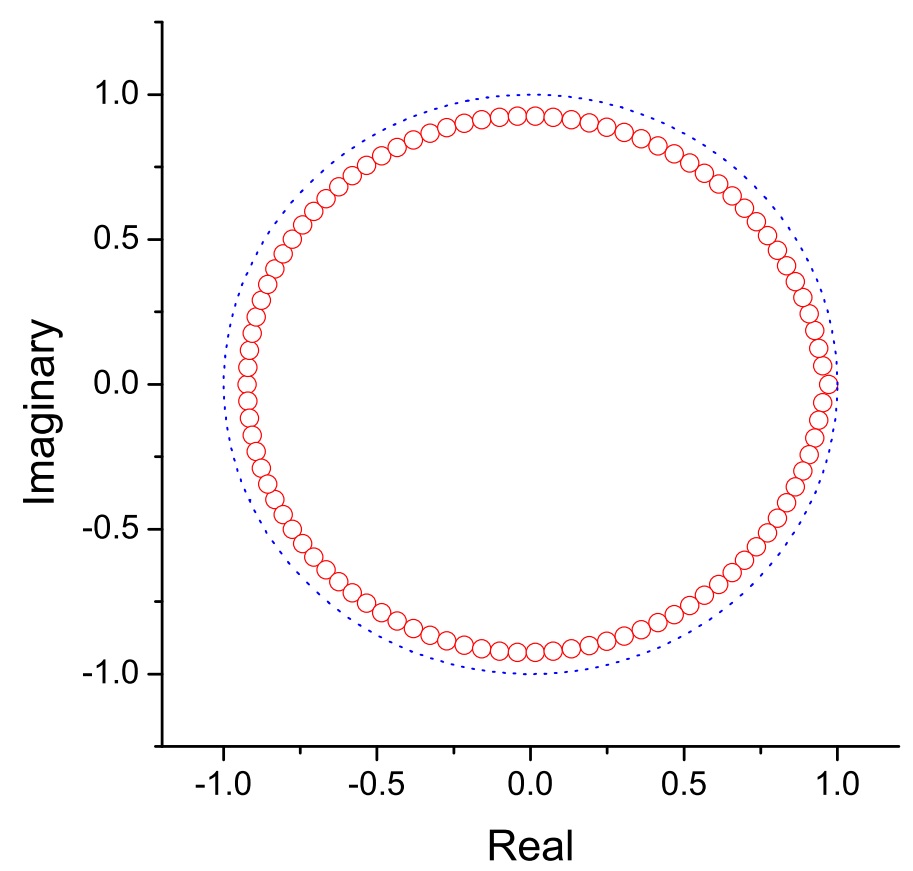

Figure 5. For a steady free-stream flow with $R e=300$, the roots of the Markov parameter polynomial (32) with $\mu=50$ and using the Markov parameters from $V_{\text {app }}$ to $C_{\mathrm{d}}$ are all contained within the unit circle.

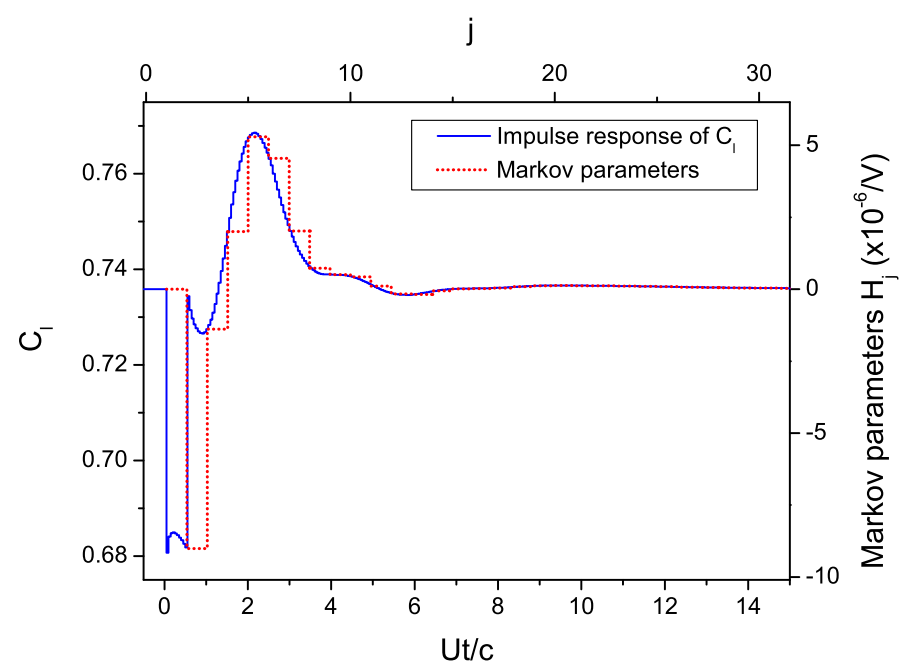

Figure 6. The Markov parameters from $V_{\text {app }}$ to $C_{1}$ are estimated from the response to a 3-kV impulse under a steady free-stream flow with $R e=300$. 


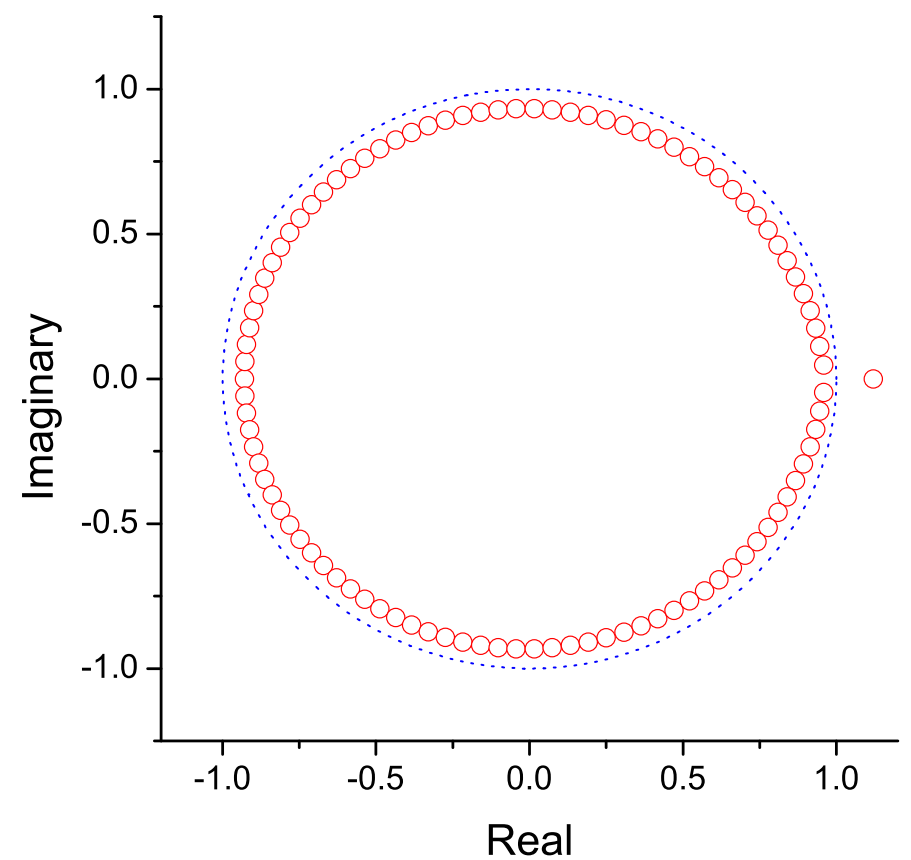

Figure 7. For a steady free-stream flow with $R e=300$, the Markov parameter polynomial (32) with $\mu=50$ and using the Markov parameters from $V_{\text {app }}$ to $C_{1}$ has one root outside of the unit circle. This root is an approximation of the nonminimum-phase zero in the linearized transfer function from $V_{\text {app }}$ to $C_{1}$.

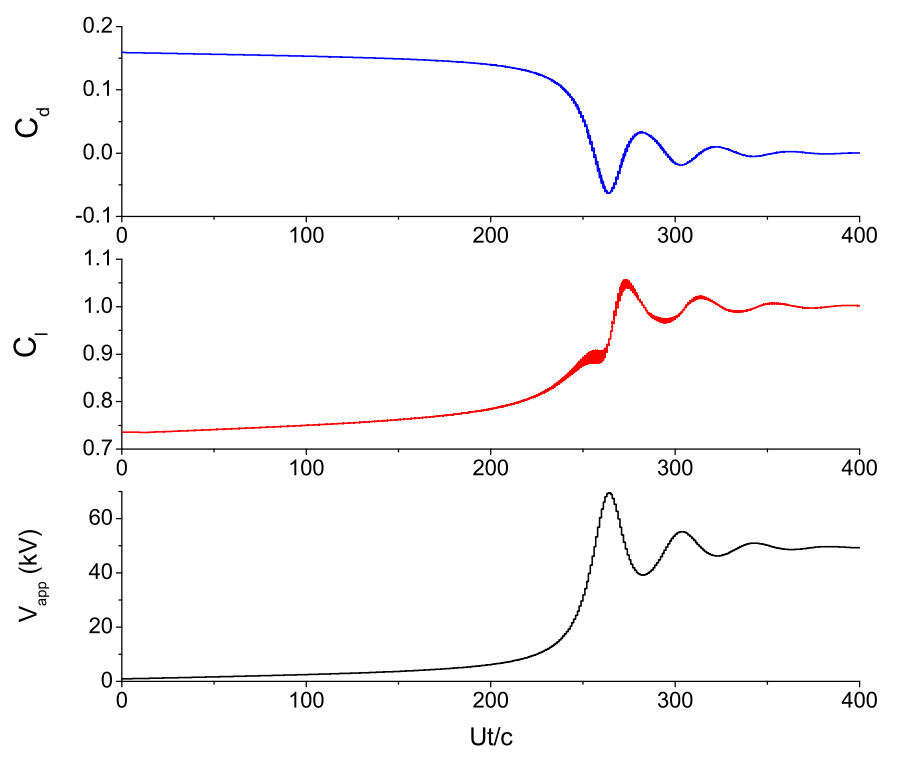

Figure 8. For a steady free-stream flow with $R e=300$, the adaptive controller (10) and (22)-(26) with $n_{\mathrm{c}}=10$ and $\bar{B}_{z u}$ constructed using 50 Markov parameters achieves the control objective of minimizing $C_{\mathrm{d}}$ by $U t / c=400$. 
state performance; however, the closed-loop converges more quickly, by $U t / c=250$. Furthermore, the peak transient performance is similar to the case where $n_{\mathrm{c}}=10$.
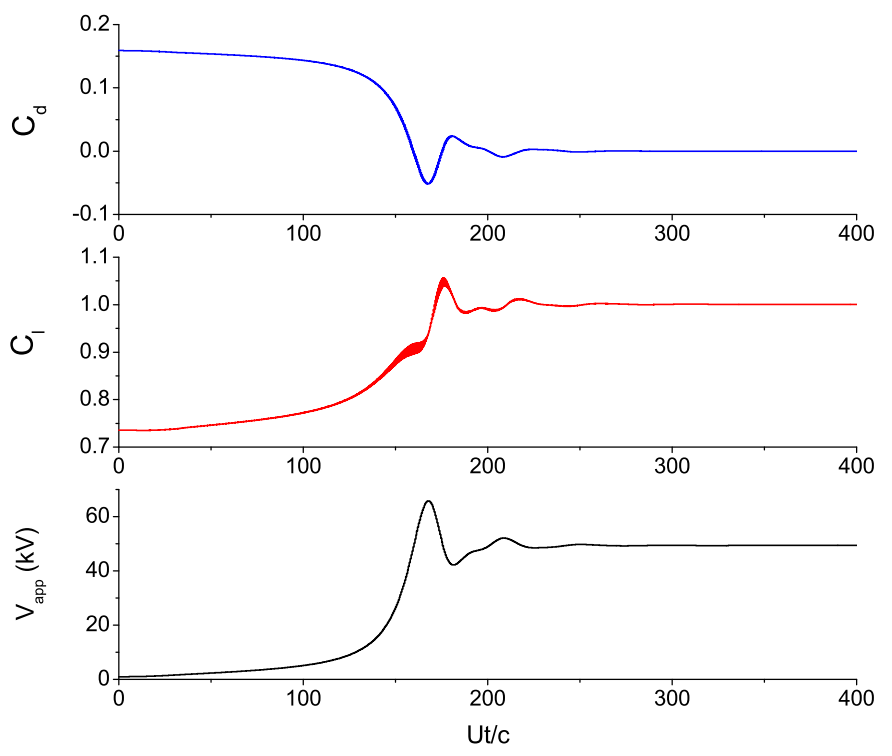

Figure 9. For a steady free-stream flow with $R e=300$, the adaptive controller (10) and (22)-(26) with $n_{\mathrm{c}}=50$ and $\bar{B}_{z u}$ constructed using 50 Markov parameters achieves the control objective of minimizing $C_{\mathrm{d}}$ by $U t / c=250$.

As we noted in the previous section, Figure 5 suggests that the linearized system from $V_{\text {app }}$ to $C_{\mathrm{d}}$ is minimum phase. As shown in Ref. 16, it is possible to control minimum phase systems using only the first non-zero Markov parameter. Figure 10 shows the closed-loop response with $n_{\mathrm{c}}=50$ and $\bar{B}_{z u}$ constructed using only the first non-zero Markov parameter. In this case, the closed-loop system converges to the same steady-state performance as shown in Figures 8 and 9; however, the closed-loop converges more quickly, by $U t / c=150$. Furthermore, the peak transient performance is similar to the cases shown in Figures 8 and 9.

\section{V.B. Minimization of $C_{\mathrm{d}}$ with sinusoidal inlet disturbance}

In this example, the control objective is to minimize the drag coefficient in the presence of an external disturbance. More specifically, the inlet velocity is disturbed from its nominal value by a sinusoid with an amplitude equal to 3 percent of the nominal free-stream speed and a period equal to 100 normalized time units. Figure 11 shows the closed-loop response with the adaptive controller (10) and (22)-(26) implemented in the feedback loop where $n_{\mathrm{c}}=50$ and $\bar{B}_{z u}$ is constructed using only the first non-zero Markov parameter from $V_{\text {app }}$ to $C_{\mathrm{d}}$. The system is allowed to run open loop until $U t / c=100$, then the adaptive controller is turned on. After approximately one period of the disturbance or by $U t / c=200, C_{\mathrm{d}}$ converges to zero.

Figure 12 shows the flow field corresponding to the steady state flow condition that is achieved in Figure 11 after $U t / c=200$. As shown in Figure 12, the adaptive control reattaches the flow and the streamlines behind the DBD actuator are flattened as compared to the nominal flow conditions shown in Figure 3.

It should be noted that while $C_{\mathrm{d}}$ is driven to zero, $C_{\mathrm{l}}$ displays steady-state oscillations. This result is somewhat surprising because there is typically a strong correlation between $C_{\mathrm{l}}$ and $C_{\mathrm{d}}$; however, the residual oscillation in $C_{1}$ can be explained by the steady-state oscillation in $V_{\text {app }}$, which occur after $U t / c=200$. The sinusoid control signal is required to cancel the impact of the sinusoidal inlet disturbance and drive $C_{\mathrm{d}}$ to zero. However, this control signal amplifies the oscillations in $C_{1}$. To explain this physically, we examine the pressure, drag, and lift coefficients at points along the surface of the airfoil at the normalized time instances $t_{1}$ and $t_{2}$, as shown in Figure 11. Figure 13 shows the value of the pressure coefficients $C_{p}$ along the surface of the airfoil at $t_{1}$ and $t_{2}$. At $t_{1}$, the control voltage $V_{\text {app }}$ is at its maximum steady-state value, whereas, at $t_{2}$, the control voltage $V_{\text {app }}$ is at its minimum steady-state value. As shown in Figure 13, when $V_{\text {app }}$ is at its maximum at $t_{1}$, the pressure on the upper surface of the airfoil near the leading edge is more negative than at $t_{2}$. Thus, the suction peak on the leading edge of the upper surface is larger at $t_{1}$ than at $t_{2}$. Similarly, 


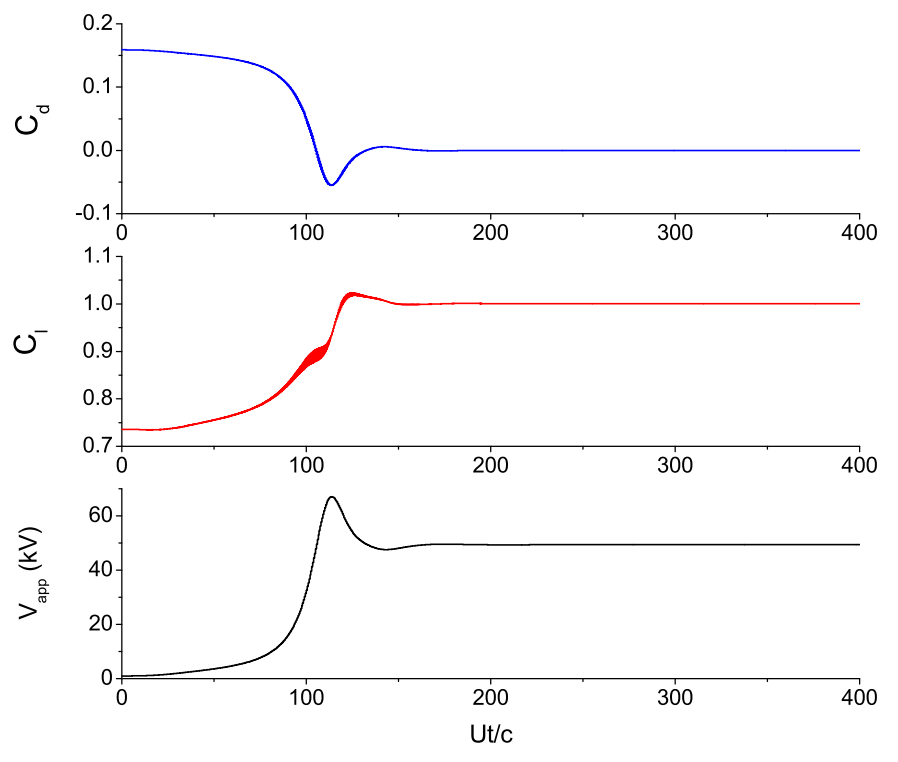

Figure 10. For a steady free-stream flow with $R e=300$, the adaptive controller (10) and (22)-(26) with $n_{\mathrm{c}}=50$ and $\bar{B}_{z u}$ constructed using only the first non-zero Markov parameter achieves the control objective of minimizing $C_{\mathrm{d}}$ by $U t / c=150$

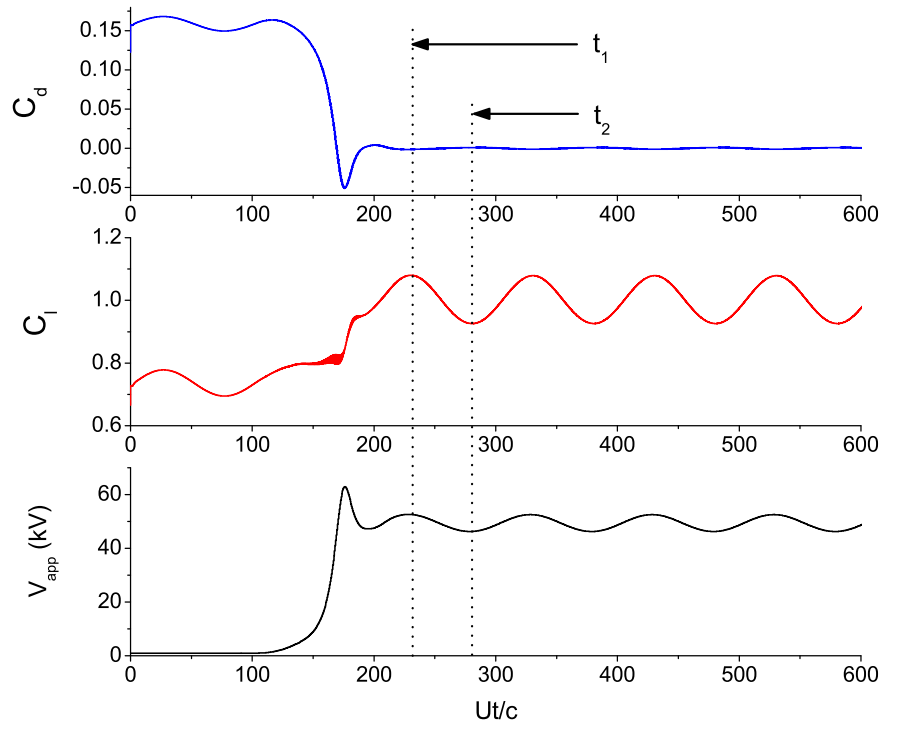

Figure 11. For a flow with sinusoidal inlet disturbance and $R e=300$, the adaptive controller (10) and (22)-(26) with $n_{\mathrm{c}}=50$ and $\bar{B}_{z u}$ constructed using only the first non-zero Markov parameter is turned on at $U t / c=100$, and the closed-loop system achieves the control objective of minimizing $C_{\mathrm{d}}$ by $U t / c=200$. 


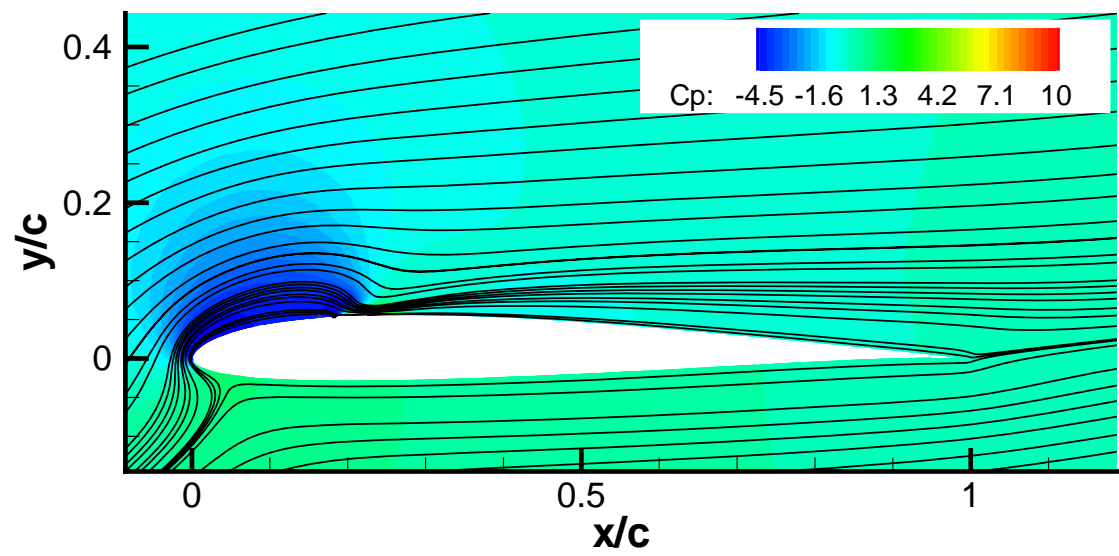

Figure 12. For a flow with sinusoidal inlet disturbance and $R e=300$, the adaptive controller (10) and (22)-(26) with $n_{\mathrm{c}}=50$ and $\bar{B}_{z u}$ constructed using only the first non-zero Markov parameter is able to reattach the flow behind the DBD actuator and flatten the streamlines.

the pressure peak located on the upper surface of the airfoil just downstream of the DBD actuator is larger at $t_{1}$ than at $t_{2}$. Thus, at $t_{1}$, the $\mathrm{DBD}$ actuator with its higher control voltage draws more flow from the upstream and induces a faster wall jet downstream when compared to the condition at $t_{2}$.

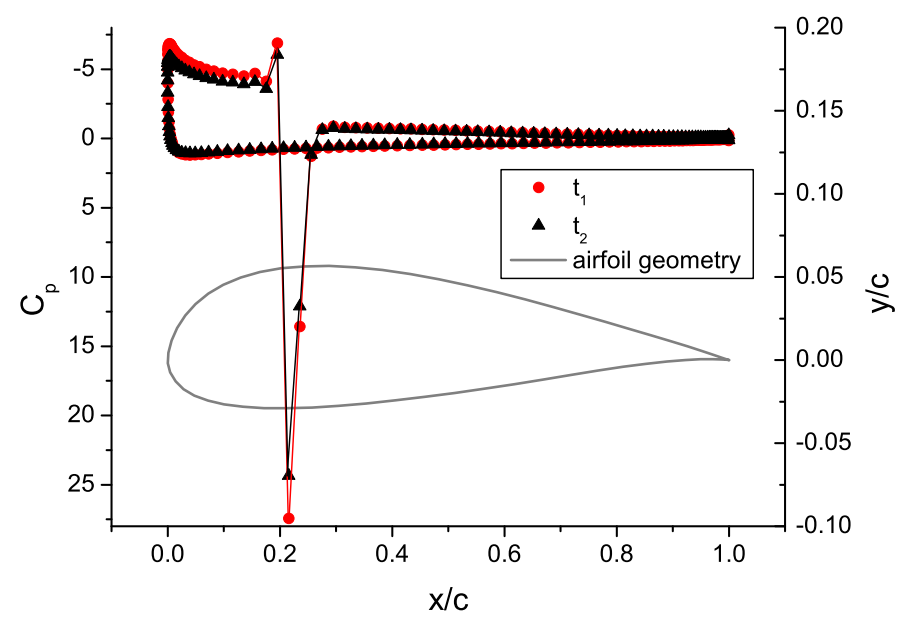

Figure 13. The suction peak on the leading edge of the upper surface of the airfoil is larger at $t_{1}$ than $t_{2}$. Similarly, the pressure peak on the upper surface of the airfoil just downstream of the DBD actuator is larger at $t_{1}$ than at $t_{2}$. Note that the flow has a sinusoidal inlet disturbance and $R e=300$.

The variation in the pressure distribution between $t_{1}$ and $t_{2}$ impacts the lift and drag forces in different ways, and thus leads to different variations in the lift and drag forces along the surface of the airfoil. Let $\hat{C}_{\mathrm{d}}(t, s)$ and $\hat{C}_{\mathrm{l}}(t, s)$ be the normalized pressure forces along the surface of the airfoil in the direction of drag and lift, respectively, as a function of normalized time $t$ and the position $s$ along the surface of the airfoil. Note that $C_{\mathrm{d}}(t)$ is the integral along the surface of the airfoil of $\hat{C}_{\mathrm{d}}(t, s)$, and $C_{\mathrm{l}}(t)$ is the integral along the surface of the airfoil of $\hat{C}_{\mathrm{l}}(t, s)$. Next, define $\delta \hat{C}_{\mathrm{d}}(s) \triangleq \hat{C}_{\mathrm{d}}\left(t_{1}, s\right)-\hat{C}_{\mathrm{d}}\left(t_{2}, s\right)$ and $\delta \hat{C}_{\mathrm{l}}(s) \triangleq \hat{C}_{\mathrm{l}}\left(t_{1}, s\right)-\hat{C}_{\mathrm{l}}\left(t_{2}, s\right)$. Figure 14 shows the value of $\delta \hat{C}_{\mathrm{d}}$ along the surface of the airfoil. The change in the suction peak between $t_{1}$ and $t_{2}$ results in a value of $\delta \hat{C}_{\mathrm{d}}$ that is negative at the leading edge and later becomes positive. The change in the pressure peak between $t_{1}$ and $t_{2}$ causes $\delta \hat{C}_{\mathrm{d}}$ to be negative. Integrating $\delta \hat{C}_{\mathrm{d}}(s)$ along the surface of the airfoil yields approximately zero because $\delta \hat{C}_{\mathrm{d}}$ is negative then positive then negative again. Thus, $C_{\mathrm{d}}\left(t_{1}\right) \approx C_{\mathrm{d}}\left(t_{2}\right)$.

In comparison, Figure 15 shows the value of $\delta \hat{C}_{1}$ along the surface of the airfoil. The change in the 


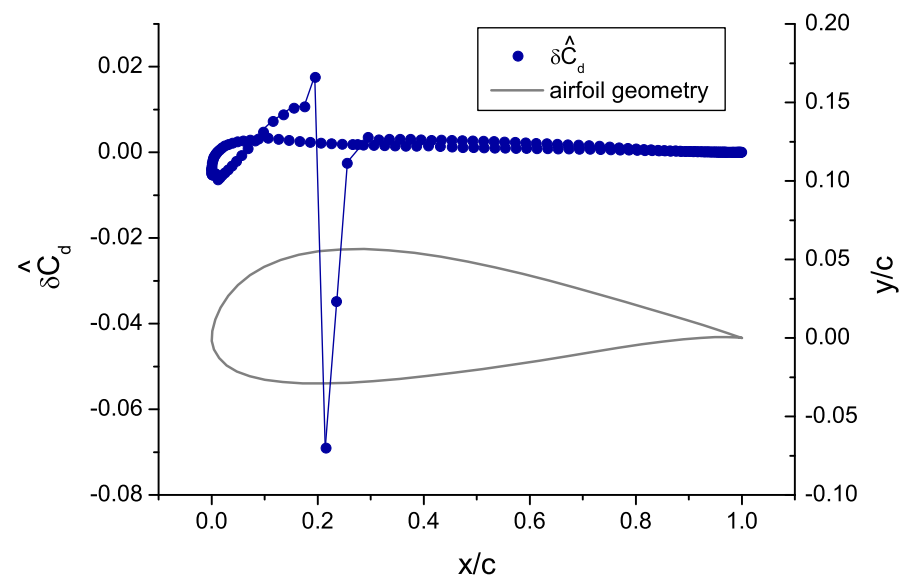

Figure 14. In the direction of the drag force, the change in the suction peak approximately cancels the change in pressure peak between $t_{1}$ and $t_{2}$. Therefore, the integral of $\delta \hat{C}_{\mathrm{d}}(s)$ along the surface of the airfoil is approximately zero, and thus, $C_{\mathrm{d}}\left(t_{1}\right) \approx C_{\mathrm{d}}\left(t_{2}\right)$. Note that the flow has a sinusoidal inlet disturbance and $R e=300$.

suction peak between $t_{1}$ and $t_{2}$ results in a value of $\delta \hat{C}_{1}$ that is positive. The change in the pressure peak between $t_{1}$ and $t_{2}$ causes $\delta \hat{C}_{1}$ to be negative. However, integrating $\delta \hat{C}_{\mathrm{d}}(s)$ along the surface of the airfoil yields a positive number because the increase in the suction peaks exceeds the decrease in the pressure peak. That is, the integral of the positive portion of $\delta \hat{C}_{1}$ exceeds the integral of the negative portion of $\delta \hat{C}_{1}$. Thus, $C_{\mathrm{d}}\left(t_{1}\right)>C_{\mathrm{d}}\left(t_{2}\right)$, as shown in Figure 11. Additional numerical study is required to determine whether it

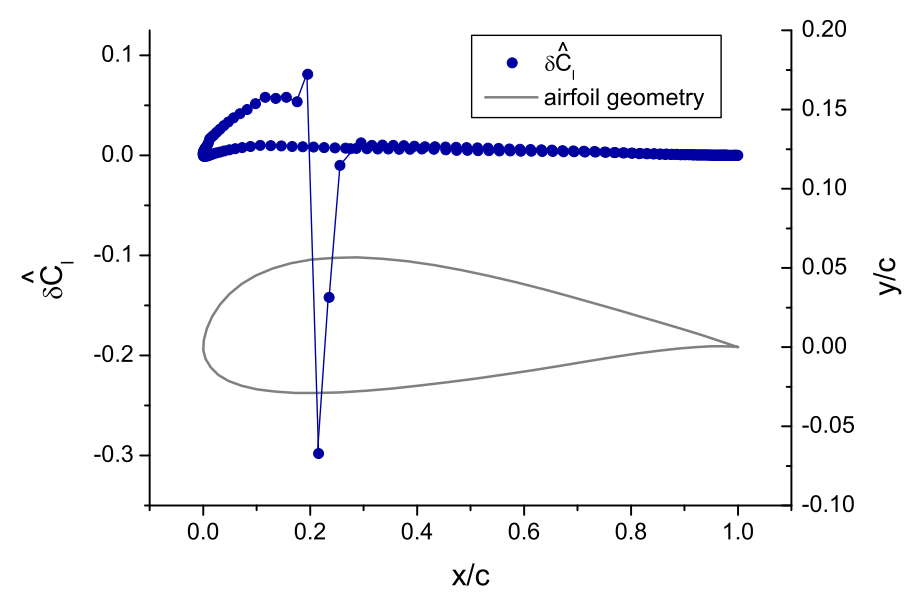

Figure 15. In the direction of the lift force, the change in the suction peak exceeds the change in the pressure peak between $t_{1}$ and $t_{2}$. Therefore, the integral of $\delta \hat{C}_{1}(s)$ along the surface of the airfoil is positive, and thus, $C_{\mathrm{d}}\left(t_{1}\right)>C_{\mathrm{d}}\left(t_{2}\right)$. Note that the flow has a sinusoidal inlet disturbance and $R e=300$.

is possible to simultaneously stabilize both $C_{\mathrm{d}}$ and $C_{\mathrm{l}}$ in the presence of an inlet disturbance with a single DBD actuator. The ability to simultaneously stabilize both $C_{\mathrm{d}}$ and $C_{\mathrm{l}}$ may depend on the airfoil geometry as well as the position and orientation of the actuator.

\section{V.C. Drive $C_{1}$ to a commanded value without inlet disturbance}

In this example, the performance variable $z=C_{1}-0.8$. Thus, the objective is to drive $C_{1}$ from its nominal value to the commanded value 0.8 . The learning rate $\alpha=500$ is selected to enforce slow adaptation. Figure 16 shows the closed-loop response with $n_{\mathrm{c}}=50$ and $\bar{B}_{z u}$ constructed using the 50 Markov parameters identified in Figure 6. Note that the time average of $C_{1}$ converges to the commanded value 0.8; however, $C_{1}$ 
oscillates with small amplitude about the commanded value. It is anticipated that these oscillations will die out over time. Alternatively, adjusting the learning rate $\alpha$ may improve the convergence speed.

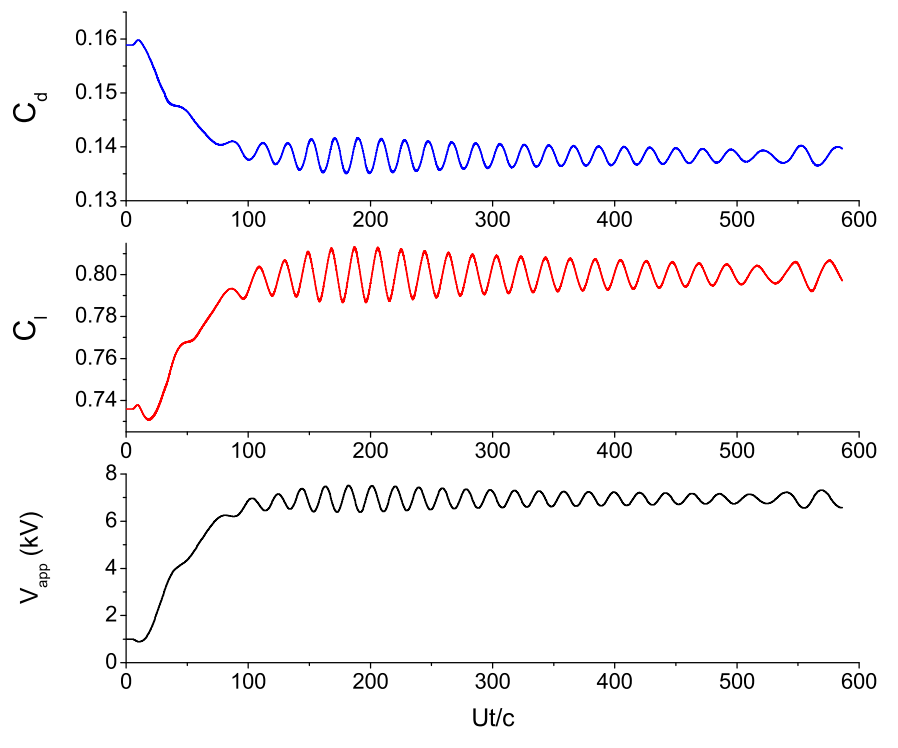

Figure 16. For a steady free-stream flow with $R e=300$, the adaptive controller (10) and (22)-(26) with $n_{\mathrm{c}}=10$ and $\bar{B}_{z u}$ constructed using 50 Markov parameters drives the time average of $C_{1}$ to the commanded value 0.8. However, there are small amplitude residual oscillations in $C_{1}$.

As noted in the previous section, Figure 7 suggests that the linearized system from $V_{\text {app }}$ to $C_{1}$ has a single nonminimum-phase zero. Numerical evidence suggests that it is possible to control nonminimumphase systems using knowledge of only the first non-zero Markov parameter and the nonminimum-phase zeros. More specifically, $\bar{B}_{z u}$ is constructed from the polynomial (32) where $\mu$ equals $d$ plus the number of nonminimum-phase zeros, $H_{d}$ is the first non-zero Markov parameter, and $H_{d+1}, \ldots, H_{\mu}$ are selected so that the roots of (32) are approximately the nonminimum-phase zeros. For this example, $\bar{B}_{z u}$ is constructed using the first non-zero Markov parameter and the approximation of the single nonminimum-phase zero given by the root in Figure 7 that lies outside of the unit circle. Figure 17 shows the closed-loop response with $n_{\mathrm{c}}=50$. The time average of $C_{1}$ converges to the commanded value 0.8 . Furthermore, the residual oscillations in $C_{1}$ are improved over the case where all 50 Markov parameters are used.

\section{V.D. Minimization of $C_{\mathrm{d}}$ with $R e=60,000$ and no inlet disturbance}

In this example, the control objective is to minimize $C_{\mathrm{d}}$ or a flow condition with $R e=60,000$, the airfoil angle of attack set to 11 degrees, and the DBD actuator is located at 5 percent of the cord length from the leading edge of the airfoil. For this example, the time step is 0.005 . We identify the first 50 Markov parameters using the impulse response method described in Section IV. The Markov parameter polynomial (32) with $\mu=50$ using the 50 identified Markov parameters has all of its roots within the unit circle. Thus, it can be inferred that the linearized discrete-time system from $V_{\text {app }}$ to $C_{\mathrm{d}}$ is minimum phase, and we construct $\bar{B}_{z u}$ using only the first non-zero Markov parameter. The learning rate $\alpha=300$ is selected to enforce slow adaptation. Figure 18 shows the closed-loop time histories for $C_{\mathrm{d}}, C_{\mathrm{l}}$, and $V_{\mathrm{app}}$ when the adaptive controller (10) and (22)-(26) is implemented in the feedback loop with $n_{\mathrm{c}}=50$. The adaptive controller is turned on at $U t / c=10$ and, by $U t / c=20, C_{\mathrm{d}}$ converges to zero. At this steady-state condition, $C_{1}=1.37$ and $V_{\text {app }}=6$ $\mathrm{kV}$. Note that the voltage required to minimize $C_{\mathrm{d}}$ in this case is significantly less than the voltage required in the case $R e=300$ because the nominal flow separation in the case $R e=60,000$ is more moderate, as shown in Figure 19. The nominal flow is mostly attached; however, there is a separation bubble near the trailing edge of the airfoil, which covers about 40 percent of the airfoil length. Figure 20 shows the flow structure is reattached after the adaptive controller minimizes the drag coefficient. 


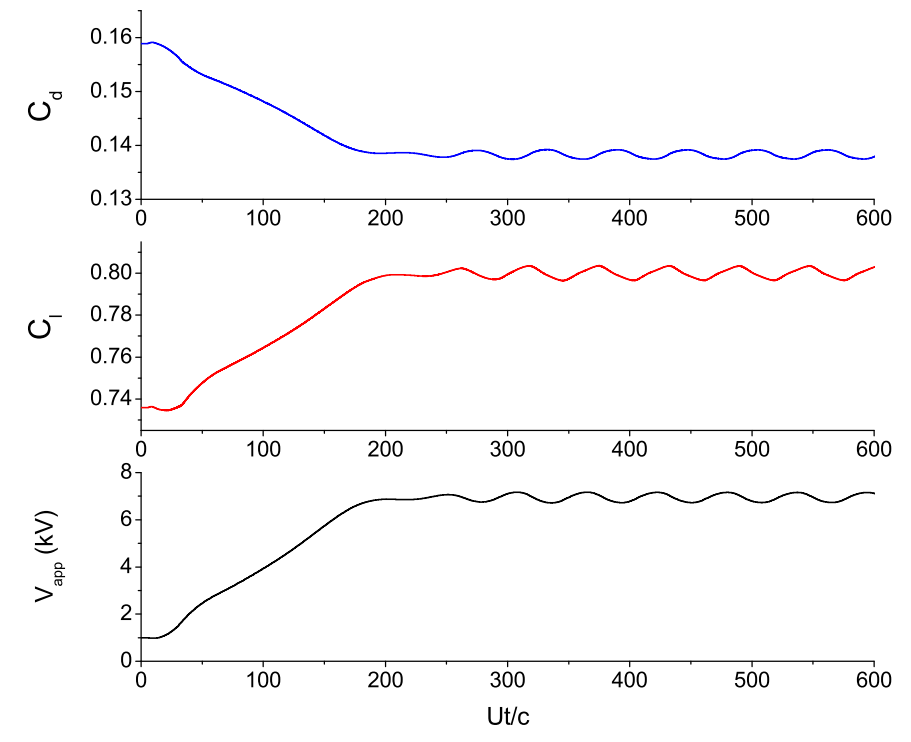

Figure 17. For a steady free-stream flow with $R e=300$, the adaptive controller (10) and (22)-(26) with $n_{\mathrm{c}}=10$ and $\bar{B}_{z u}$ constructed using only the first non-zero Markov parameter and the single nonminimum-phase zero drives the time average of $C_{1}$ to the commanded value 0.8. Furthermore, the amplitude of the residual oscillations in $C_{1}$ is smaller than in the case where all 50 Markov parameters are used (see Figure 16).

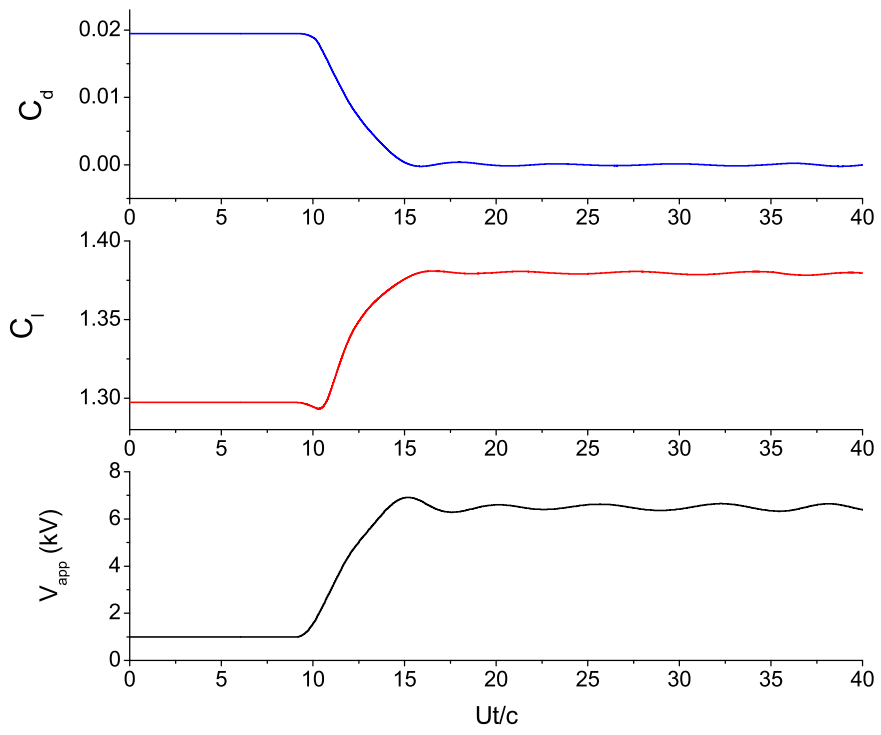

Figure 18. For a steady free-stream flow with $R e=60,000$, the adaptive controller (10) and (22)-(26) with $n_{\mathrm{c}}=50$ and $\bar{B}_{z u}$ constructed using only the first non-zero Markov parameter is turned on at $U t / c=10$ and achieves the control objective of minimizing $C_{\mathrm{d}}$ by $U t / c=20$. 


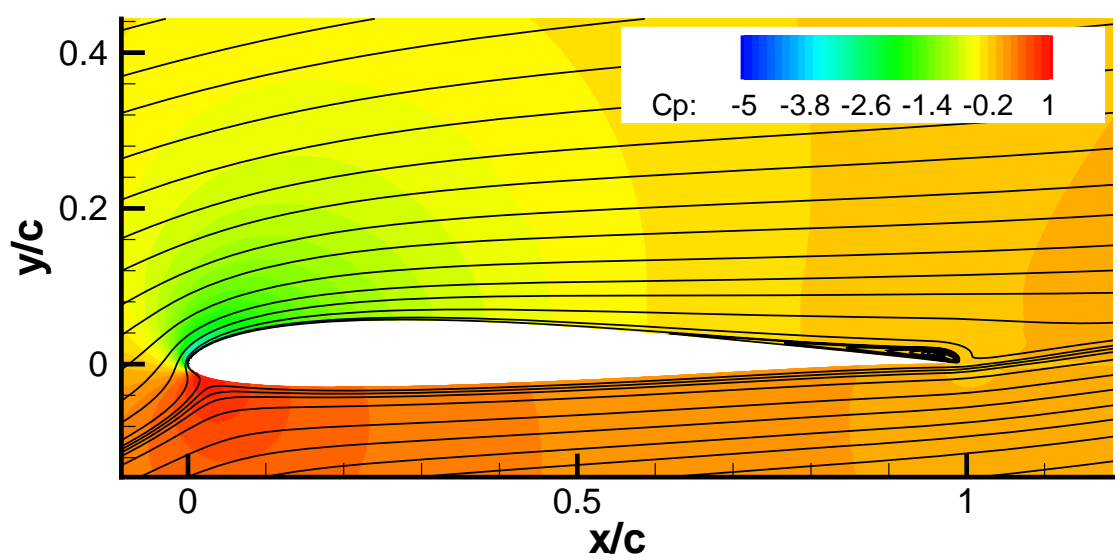

Figure 19. For a steady free-stream flow with $R e=60,000$, a $1 \mathrm{kV}$ nominal DBD actuator voltage results in a separation bubble near the trailing edge of the airfoil.

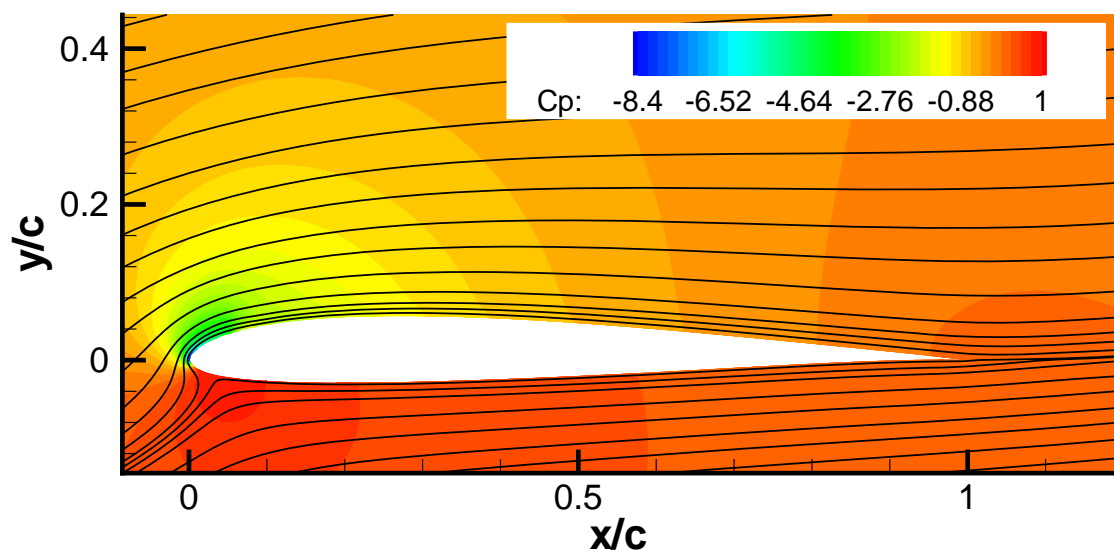

Figure 20. For a steady free-stream flow with $R e=60,000$, the adaptive controller (10) and (22)-(26) with $n_{\mathrm{c}}=50$ and $\bar{B}_{z u}$ constructed using only the first non-zero Markov parameter is able to reattach the flow behind the DBD actuator. 


\section{Conclusions}

In this paper, we used a discrete-time adaptive controller to control the aerodynamics around a SD 7003 airfoil with a DBD actuator located near the leading edge of the airfoil. More specifically, numerical flow simulations demonstrated that the adaptive control is able to minimize the airfoil's drag coefficient $C_{\mathrm{d}}$ for both steady and sinusoidally disturbed free-stream flows with a Reynolds number of 300 . In addition, the adaptive controller was able to minimize $C_{\mathrm{d}}$ for steady free stream flows with a Reynolds number of 60,000 . In these cases, the adaptive controller was able to achieve the control objective using very limited information of the aerodynamics. In particular, the adaptive controller requires only knowledge of the relative degree $d$ and the first non-zero Markov parameter $H_{d}$ of the linearized plant dynamics from $V_{\text {app }}$ to $C_{\mathrm{d}}$. The adaptive algorithm is able to minimize $C_{\mathrm{d}}$ with limited plant information because, in part, the linearized dynamics from $V_{\text {app }}$ to $C_{\mathrm{d}}$ appear to be minimum phase. However, the adaptive controller has been shown numerically to work well on nonminimum-phase systems as well. In this paper, the adaptive control was demonstrated on the dynamics from $V_{\text {app }}$ to $C_{\mathrm{l}}$, which was shown to be nonminimum phase. In this case, the adaptive controller successfully drove $C_{1}$ to a positive commanded value using limited plant information, namely knowledge of $d, H_{d}$, and the location of the single nonminimum-phase zero in the linearized transfer function from $V_{\text {app }}$ to $C_{1}$. Future work will include more extensive numerical study of the adaptive controller's performance under different flow conditions, including a wider range of Reynolds numbers and free-stream flow conditions. In addition, further work is required to determine if it is feasible for the adaptive controller to simultaneous stabilize $C_{\mathrm{d}}$ and $C_{\mathrm{l}}$ under unsteady free-stream flow conditions.

\section{Acknowledgements}

The present work is supported by the Air Force Research Laboratory, under a collaborative center agreement, and by NASA grant NNX08AB92A.

\section{References}

${ }^{1}$ Lissaman, P., "Low-Reynolds-number airfoils," Ann. Rev. Fluid Mech., Vol. 15, 1983, pp. 223-239.

${ }^{2}$ Shyy, W., Lian, Y., Tang, J., Viieru, D., and Liu, H., Aerodynamics of Low Reynolds Number Flyers, Cambridge Univ. Press, New York, 2008.

${ }^{3}$ Huang, X., Chang, S., Zhang, X., and Gabriel, S., "Variable structure model for flow-induced tonal noise control with plasma actuators," AIAA Journal, Vol. 46, No. 1, January 2008, pp. 241-250.

${ }^{4}$ Muse, J., Kutay, A., and Calise, A., "Experimental trapped vorticity flight control using an augmenting error minimization adaptive law," AIAA Guidance, Navigation and Control Conference and Exhibit, Honolulu, HI, August 2008, AIAA-2008-6962.

${ }^{5}$ Rowley, C., Ahuja, S., Taira, K., and Colonius, T., "Closed-loop control of leading edge vorticity on a 3D wing: simulations and low-dimensional models," 38th Fluid Dynamics Conference and Exhibit, Seattle, WA, June 2008, AIAA-2008-3981.

${ }^{6}$ Caraballo, E., Kasnakoglu, C., Serrani, A., and Samimy, M., "Control input separation methods for reduced-order model-based feedback flow control," AIAA Journal, Vol. 46, no. 9, September 2008, pp. 2306-2322.

${ }^{7}$ King, R., Petz, R., and Lemke, O., "Adaptive flow control using slope seeking," Proc. of IEEE 14th Mediterranean Conf. on Contr. and Auto., Ancona, Italy, June 2006.

${ }^{8}$ Becker, R., King, R., Petz, R., and Nitsche, W., "Adaptive closed-loop separation control on a high-lift conguration using extremum seeking," Proc. of 3rd AIAA Flow Contr. Conf., San Francisco, CA, June 2006.

${ }^{9}$ Rizzo, M., Santillo, M., Padthe, A., Hoagg, J. B., Akhtar, S., and Bernstein, D. S., "CFD-Based Identification for Adaptive Flow Control Using ARMARKOV Disturbance Rejection," Proc. Amer. Contr. Conf., Minneapolis, MN, June 2006, pp. 3783-3788.

${ }^{10}$ Santillo, M. A., Hoagg, J. B., and Bernstein, D. S., "CFD-based adaptive flow control for steady flow field modification," Proc. Conf. Dec. Contr., San Diego, CA, December 2006, pp. 3106-3110.

${ }^{11}$ Santillo, M. A., Hoagg, J. B., Bernstein, D. S., and Powell, K., "Adaptive Disturbance Rejection for Flow in a Duct with Time-Varying Upstream Velocity,” Proc. Amer. Contr. Conf., New York, NY, July 2007, pp. 2226-2231.

${ }^{12}$ Narendra, K. S. and Annaswamy, A. M., Stable Adaptive Systems, Prentice Hall, Englewood Cliffs, New Jersey, 1989.

${ }^{13}$ Goodwin, G. C., Ramadge, P. J., and Caines, P. E., "Discrete-time multivariable adaptive control," IEEE Trans. Autom. Contr., Vol. 25, 1980, pp. 449-456.

${ }^{14}$ Rohrs, C., Valavani, L., Athans, M., and Stein, G., "Robustness of continuous-time adaptive control algorithms in the presence of unmodeled dynamics," IEEE TAC, Vol. 30, 1985, pp. 881-889.

${ }^{15}$ Anderson, B., "Topical Problems of Adaptive Control," Proc. European Contr. Conf., Kos, Greece, July 2007 , pp. 4997-4998.

${ }^{16}$ Hoagg, J. B., Santillo, M. A., and Bernstein, D. S., "Discrete-Time Adaptive Command Following and Disturbance Rejection with Unknown Exogenous Dynamics," IEEE Trans. Autom. Contr., Vol. 53, 2008, pp. 912-928.

${ }^{17}$ Venugopal, R. and Bernstein, D. S., "Adaptive disturbance rejection using ARMARKOV/Toeplitz Models," IEEE Trans. Contr. Sys. Tech., Vol. 8, 2000, pp. 257-269. 
${ }^{18}$ Cho, Y., Fledderjohn, M., Holzel, M., Jayaraman, B., Santillo, M., Bernstein, D. S., and Shyy, W., “Adaptive flow control of low reynolds number aerodynamics using a dielectric barrier discharge actuator," 47th AIAA Aerospace Sciences Meeting including the New Horizons Forum and Aerospace Exposition, Orlando, FL, January 2009, AIAA-2009-378.

${ }^{19}$ Santillo, M. A. and Bernstein, D. S., "Inherent Robustness of Minimal Modeling Discrete-Time Adaptive Control to Flight Anomalies," Proc. Guid. Nav. Contr. Conf., Honolulu, HI, August 2008, AIAA-2008-7289.

${ }^{20}$ Santillo, M. A. and Bernstein, D. S., "A Retrospective Correction Filter for Discrete-time Adaptive Control of Nonminimum Phase Systems," Proc. Conf. Dec. Contr., Cancun, Mexico, December 2008.

${ }^{21}$ M. Forte, J. J., Moreau, E., and Touchard, G., "Optimization of a dielectric barrier discharge actuator by stationary and non-stationary measurements of the induced flow velocity - application to airflow control," 3rd AIAA Flow Control Conference, San Francisco, CA, June 2006.

${ }^{22}$ Abe, T., Takizawa, Y., Sato, S., and Kimura, N., "A parametric experimental study for momentum transfer by plasma actuator," Proc. of 45th AIAA Aerospace Sciences Meeting and Exhibit, Reno, NV, January 2007, AIAA-2007-187.

${ }^{23}$ Roth, J. and Dai, X., "Optimization of the aerodynamic plasma actuator as an electrohydrodynamic (EHD) electrical device," Proc. of 44th AIAA Aerospace Sciences Meeting and Exhibit, Reno, NV, January 2006, AIAA-2006-1203.

${ }^{24}$ Cho, Y., Jayaraman, B., Viana, F., Haftka, R., and Shyy, W., "Surrogate Modeling for Characterizing the Performance of Dielectric Barrier Discharge Plasma Actuator," Proc. of 46th AIAA Aerospace Sciences Meeting and Exhibit, Reno, NV, January 2008, AIAA-2008-1381.

${ }^{25}$ Kamakoti, R., Thakur, S., Wright, J., and Shyy, W., "Validation of a new parallel all-speed CFD code in a rule-based framework for multidisciplinary applications," Proc. of 3rd AIAA Flow Contr. Conf, June 2006, AIAA-2006-3063.

${ }^{26}$ Menter, F., "Two-equation eddy-viscosity turbulence models for engineering applications," AIAA Journal, Vol. 32, No. 8, 1994, pp. 1598-1605.

${ }^{27}$ Shyy, W., Jayaraman, B., and Anderson, A., "Modeling of glow-discharge induced flow dynamics," J. Appl. Phys., Vol. 92(11), 2002, pp. 6434-6443.

${ }^{28}$ Jayaraman, B., Lian, Y., and Shyy, W., "Low-reynolds number flow control using dielectric barrier discharge actuators," 37th AIAA Fluid Dynamics Conference and Exhibit, Miami, FL, June 2007, AIAA paper 2007-3974.

${ }^{29}$ Grundmann, S., Klumpp, S., and Tropea, C., "Experimental and numerical investigations of boundary-lay influence using plasma-actuators," Active Flow Control, NNFM 95, New York: Springer, 2007, pp. 56-68.

${ }^{30}$ Gherardi, N. and Massine, F., "Mechanisms controlling the transition from glow silent discharge to streamer discharge in nitrogen," IEEE Trans. Plasma Sci., Vol. 29, No. 3, 2001, pp. 536-544.

${ }^{31}$ Kekez, M. M., Barrault, M. R., and Craggs, J. D., "Spark channel formation," J. Phys. D: Appl. Phys., Vol. 3, No. 12, 1970, pp. 1886-1896.

${ }^{32}$ Moreau, E., "Airflow control by non-thermal plasma actuators," J. Phys. D: Appl. Phys., Vol. 40, No. 3, 2007, pp. 605636. 